\title{
Relación entre agilidad y capacidades coordinativas en niños futbolistas ${ }^{*}$
}

\author{
Luis Yesid Viancha Amaya** \\ Paulo Jonathan Acosta Tova ${ }^{* * *}$
}

Recibido: noviembre 7 de 2017 • Aceptado: marzo 8 de 2018

\section{Resumen}

El presente artículo tuvo como objetivo determinar la relación entre la agilidad y las capacidades coordinativas en niños de la Escuela de Formación Deportiva del Instituto Municipal de Deporte y Recreación de Tunja IRDET. Para esto, 30 jugadores de género masculino fueron intervenidos, con edades comprendidas entre los 12 y los 14 años. Las capacidades de coordinación fueron evaluadas con el test motor de Caminero y la agilidad con el test de Illinois. De manera grupal, en referencia a la agilidad, los participantes presentaron un tiempo promedio de $18.4223 \pm 1.1779$ segundos, y en lo referente a la capacidad coordinativa, los deportistas mostraron un tiempo promedio de $18.9037 \pm 1.59467$ segundos. Se concluyó que existe una relación no significativa, débil y directa entre la agilidad y

" Artículo de investigación con financiación propia. Universidad Pedagógica y Tecnológica de Colombia (UPTC), Tunja, Colombia. Citar como: Viancha, A. y Acosta, T. (2018). Relación entre agilidad y capacidades coordinativas en niños futbolistas. Cuerpo, Cultura y Movimiento, 8(1), 13-26. DOI: https://doi.org/10.15332/2422474X.5119

** Docente Bienestar Universitario, Universidad Santo Tomás, Tunja, Colombia. Correo electrónico: Luis Viancha, correo deporte@samaca-boyaca.gov.co / ORCID: https:// orcid.org/0000-0002-7672-9442

***Docente Universidad Pedagógica y Tecnológica de Colombia (UPTC), Tunja, Colombia. Correo electrónico: paulo.acosta@uptc.edu.co / ORCID: https://orcid. org/0000-0002-7718-7235 
las capacidades coordinativas, lo cual indica que un aumento en la agilidad implica un incremento débil en las capacidades coordinativas y viceversa.

Palabras clave: agilidad, capacidades coordinativas, test motor de Caminero, test de Illinois. 


\section{Relationship between agility and coordinating capacities in football players}

\section{Abstract}

The objective of this article was to determine the relationship between agility and coordination skills in children of the Sports Training School of the Municipal Sports and Recreation Institute of Tunja - IRDET. For this, 30 male players were intervened, with ages between 12 and 14 years. The coordination skills were evaluated with the Caminero motor test and the agility with the Illinois test. As a group, in reference to agility, the participants presented an average time of $18.4223 \pm 1.1779$ seconds, and as regards the coordinative capacity, the participants showed an average time of 18.9037 \pm 1.59467 seconds. It was concluded that there is a non-significant, weak and direct relationship between agility and coordinative capacities, which indicates that an increase in agility implies a weak increase in coordinative capacities and vice versa.

Keywords: agility, coordinative abilities, Caminero motor test, Illinois test. 


\section{Introducción}

En el fútbol el proceso de enseñanza-aprendizaje de la agilidad y las capacidades coordinativas, que debe estar implícito dentro de los diseños de los programas para el trabajo multilateral en edades tempranas, en muchas ocasiones ha sido o muy poco o nada aplicado. En el trabajo o desarrollo de un jugador de fútbol, se requiere la exigencia de muchos factores, que, si no se trabajan en las edades tempranas, serán elementos que actuarían como un lastre negativo en el desarrollo futuro del futbolista, en cualquiera de las posiciones en el campo de juego (Pérez, 2016).

Según Caminero (2009), las capacidades coordinativas se pueden definir como el "conjunto de capacidades que organizan y regulan de forma precisa todos los procesos parciales de un acto motor en función de un objetivo motor preestablecido", y la agilidad como una cualidad compleja que le permite a un atleta reaccionar a un estímulo, comenzar a moverse de forma rápida y eficiente, avanzar en la dirección correcta, y estar dispuesto a cambiar de dirección o detenerse rápidamente para hacer una técnica deportiva de una forma rápida, eficiente y de manera reproducible (Young y Farrow, 2006).

El desarrollo de las capacidades coordinativas se manifiesta de forma diferente, por lo que independientemente de que el deporte sea colectivo, su práctica debe ser individual; por ende, hay que buscar desarrollar una relación mayor entre los componentes del proceso coordinativo (Pérez, 2016).

Es así como el fútbol propone situaciones y movimientos de juego con los cuales se pueden evidenciar diferentes acciones, es por esto que se toma el fútbol en la categoría infantil, que es donde se forma la base primordial al rendimiento. En este sentido, el interés es indagar en aras de demostrar la posible la relación entre las variables agilidad y las capacidades coordinativas en los niños.

\section{Metodología}

La población corresponde a 30 jugadores de la rama masculina de la Escuela de Formación Deportiva del Instituto Municipal de Deporte y Recreación de Tunja - IRDET, con edades promedio de $13.07 \pm 0.828$ años, estatura promedio de $1.4507 \pm 0.07273$ metros y con un peso promedio de 49.7667 
\pm 7.56436 kilogramos. Todos los participantes fueron informados en detalle acerca de los procedimientos experimentales y los posibles riesgos y beneficios de esta investigación. El estudio obtuvo el asentimiento informado por escrito de todos los atletas antes de comenzar la fase experimental, autorizado por los acudientes de cada uno de los deportistas.

Para la realización de esta investigación, los instrumentos utilizados fueron el test de illinois y el test motor de Caminero para evaluar la agilidad y las capacidades coordinativas, respectivamente; por todo ello, el objetivo de este estudio fue determinar la relación entre la agilidad y las capacidades coordinativas en niños, y luego se realizó el análisis e interpretación de resultados para observar si existe relación entre las variables planteadas.

La clasificación de la agilidad se codificó de la siguiente manera:

1. Promedio excelente, para tiempos inferiores a 15.2 segundos.

2. Sobre promedio, para tiempos entre 15.2 y 16.1 segundos.

3. Promedio, para tiempos entre 16.2 y 18.1 segundos.

4. Promedio bajo, para tiempos entre 18.2 y 18.8 segundos.

5. Promedio pobre, para tiempos superiores a 18.8 segundos.

En las capacidades coordinativas, los ítems de calificación fueron:

1. Extremadamente bueno, para tiempos inferiores a 17.75 segundos.

2. Muy bueno, para tiempos entre 17.753 y 18.85 segundos.

3. Bueno, para tiempos entre 18.859 y 21.22 segundos.

4. Bueno sobre la media, para tiempos entre 21.227 y 25.17 segundos.

5. Pobre bajo la media, para tiempos entre 25.175 y 29.12 segundos.

6. Pobre, para tiempos entre 29.122 y 31.49 segundos.

7. Muy pobre, para tiempos entre 31.491 y 32.59 segundos.

8. Extremadamente pobre, para tiempos superiores a 32.59 segundos. 


\section{Resultados y discusión}

El procesamiento de los datos se realizó mediante el software estadístico SPSS 13 en su versión estudiantil, inicialmente se hizo un análisis exploratorio de datos mediante estadística descriptiva, luego se efectuó un análisis descriptivo tanto de la agilidad como de la capacidad coordinativa y por último, se efectuó un análisis de correlación entre la agilidad y la capacidad coordinativa que involucró pruebas de significancia.

De manera grupal, en referencia a la agilidad, los participantes presentaron un tiempo promedio de $18.4223 \pm 1.1779$ segundos, donde la cantidad 1.1779 es la desviación estándar (tabla 1). El CV es 1.1779/18.4223 =0.0639 $=6.39 \%$, este valor indica que los participantes son homogéneos (similares) con respecto a la agilidad.

Tabla 1. Tiempo promedio para valorar la agilidad de los participantes

\begin{tabular}{ccc}
\hline $\mathrm{N}$ & Valid & 30 \\
& Missing & 0 \\
\hline Mean & & 18.4223 \\
Std. Deviation & & 1.17790 \\
Minimum & 15.36 \\
Maximum & 22.15 \\
\hline
\end{tabular}

Fuente: elaboración propia.

En concordancia con la clasificación generada a través del test de Illinois, ninguno de los participantes logró el nivel de excelente; los individuos se clasificaron de la siguiente manera: el $3.3 \%$ alcanzó el nivel de sobre promedio, el $40 \%$ se ubicó en el nivel de promedio, un $23.3 \%$ estuvo en el nivel de bajo promedio y el $33.3 \%$ restante en un nivel pobre con respecto a la agilidad. Estos resultados permiten afirmar que un alto porcentaje $(96.7 \%)$ de los participantes alcanzó a estar en el promedio o por debajo de este (tabla 2); por lo tanto, en general el grupo puede clasificarse en el nivel de promedio bajo. 
Tabla 2. Clasificación por niveles de agilidad

\begin{tabular}{llcccc}
\hline $\begin{array}{l}\text { Niveles de } \\
\text { agilidad }\end{array}$ & Frequency & Percent & $\begin{array}{c}\text { Valid } \\
\text { Percent }\end{array}$ & $\begin{array}{c}\text { Cumulative } \\
\text { Percent }\end{array}$ \\
\hline Valid & $\begin{array}{l}\text { Sobre } \\
\text { promedio }\end{array}$ & 1 & 3.3 & 3.3 & 3.3 \\
& $\begin{array}{l}\text { Promedio } \\
\text { Bajo }\end{array}$ & 12 & 40.0 & 40.0 & 43.3 \\
& $\begin{array}{l}\text { promedio } \\
\text { Pobre }\end{array}$ & 10 & 23.3 & 23.3 & 66.7 \\
& Total & 30 & 100.0 & 100.0 & \\
\hline
\end{tabular}

Fuente: elaboración propia.

De forma grupal, en lo referente a las capacidades coordinativas (tabla 3), los participantes presentaron un tiempo promedio de $18.9037 \pm$ 1.59467 segundos, donde la cantidad 1.59467 es la desviación estándar. El CV $=1.59467 / 18.9037=0.0843=8.43 \%$, este valor evidencia que los participantes resultaron homogéneos (semejantes) con respecto a la capacidad coordinativa.

Tabla 3. Tiempo promedio para valorar la capacidad coordinativa de los participantes

\begin{tabular}{ccc}
\hline $\mathrm{N}$ & Valid & 30 \\
& Missing & 0 \\
\hline Mean & & 18.9037 \\
Std. Deviation & 1.59467 \\
Minimum & 17.23 \\
Maximum & 24.17 \\
\hline
\end{tabular}

Fuente: elaboración propia.

En correspondencia con la clasificación originada por medio del test de Caminero (tabla 4), el $20 \%$ logró el nivel extremadamente bueno, el $43.3 \%$ alcanzó el nivel de muy bueno, el 30 \% consiguió el nivel de bueno 
y el $6.7 \%$ se ubicó en el nivel de bueno sobre la media. Estos resultados permiten afirmar que un alto porcentaje $(100 \%)$ de los participantes alcanzó a clasificarse en el nivel de bueno sobre la media, bueno, muy bueno o extremadamente bueno. En general, el grupo puede clasificarse en el nivel de bueno.

Tabla 4. Clasificación por niveles de capacidad coordinativa

\begin{tabular}{llcccc}
\hline $\begin{array}{l}\text { Niveles de } \\
\text { capacidad } \\
\text { coordinativa }\end{array}$ & Frequency & Percent & $\begin{array}{c}\text { Valid } \\
\text { Percent }\end{array}$ & $\begin{array}{c}\text { Cumulative } \\
\text { Percent }\end{array}$ \\
\hline Valid & $\begin{array}{l}\text { Extremada- } \\
\text { mente bueno }\end{array}$ & 6 & 20.0 & 20.0 & 20.0 \\
& Muy bueno & 13 & 43.3 & 43.3 & 63.3 \\
& Bueno & 9 & 30.0 & 30.0 & 93.3 \\
& $\begin{array}{l}\text { Bueno sobre } \\
\text { la media }\end{array}$ & 12 & 6.7 & 6.7 & 100.0 \\
& Total & 30 & 100.0 & 100.0 & \\
\hline
\end{tabular}

Fuente: elaboración propia.

Con el propósito de analizar la relación existente entre la agilidad y las capacidades coordinativas, o lo que es lo mismo, la correlación entre estos dos aspectos, se obtuvo la matriz de correlaciones de Pearson. Las interpretaciones e inferencias se realizaron con un nivel de significancia del $5 \%(0.05)$.

De los resultados obtenidos (tabla 5), se deduce que existe una correlación no significativa al $5 \%(0.05)$ entre la agilidad y la capacidad coordinativa debido a que se obtuvo un valor_p $=0.815$, el cual es superior a 0.05; el coeficiente de correlación de Pearson fue de $r=0.045, \mathrm{p}<0.05$, 2_colas (2-tailed). En consecuencia, al interpretar el coeficiente se concluyó que existe una relación débil y directa entre la agilidad y la capacidad coordinativa, la cual resultó ser no significativa. Lo anterior puede interpretarse como que un aumento en la agilidad implica un incremento débil (pequeño) en la capacidad coordinativa y viceversa, un incremento en la capacidad coordinativa de los participantes genera un aumento débil en su agilidad. 
Tabla 5. Matriz de correlaciones entre la agilidad y la capacidad coordinativa

\begin{tabular}{cccc}
\hline & & Agilidad & Capacidad coordinativa \\
\hline Agilidad & Pearson Correlation & 1 & 0.045 \\
& Sig. (2-tailed) & & 0.815 \\
& $\mathrm{~N}$ & 30 & 30 \\
Capacidad & Pearson Correlation & 0.045 & 1 \\
coordinativa & Sig. (2-tailed) & 0.815 & 30 \\
\hline $\mathrm{N}$ & & 30 &
\end{tabular}

Fuente: elaboración propia.

Se estableció si la relación entre la capacidad coordinativa y la agilidad era significativa mediante una regresión lineal y su correspondiente análisis de varianza (tabla 6). De allí se dedujo que la prueba no es significativa ya que su valor_p (Sig.) $=0.815$ es superior al 0.05 . En consecuencia, la relación entre la capacidad coordinativa y la agilidad es no significativa.

Tabla 6. Análisis de varianza de la correlación entre la capacidad coordinativa y la agilidad

\begin{tabular}{llccccc}
\hline Modelo & & $\begin{array}{c}\text { Sum. of } \\
\text { Squares }\end{array}$ & Df & $\begin{array}{c}\text { Mean } \\
\text { Square }\end{array}$ & F & Sig. \\
\hline 1 & Regression & 0.147 & 1 & 0.147 & 0.056 & $0.815(\mathrm{a})$ \\
& Residual & 73.599 & 28 & 2.629 & & \\
\hline & Total & 73.746 & 29 & & & \\
\hline
\end{tabular}

Fuente: elaboración propia.

Por otra parte, se estableció que el modelo de regresión lineal que explica la capacidad coordinativa a través de la agilidad, es $\mathrm{y}=0.059 \mathrm{x}+$ 17.809 , donde la variable y corresponde a la capacidad coordinativa y la variable x corresponde a la agilidad. En este análisis (tabla 7), se deduce que el coeficiente de $\mathrm{x}$ es no significativo debido a que su valor_p (Sig.) $=0.815$ es superior a 0.05; además, el término independiente de 17.809 sí resultó significativo, ya que su p_valor (Sig.) $=0.001$ es inferior al 0.05 . Los puntos 
que relacionan la capacidad coordinativa con la agilidad no siguen una determinada línea de tendencia; es decir, los puntos guardan una correlación no significativa ya que esta corresponde al coeficiente de correlación de Pearson $\mathrm{r}=0.045$.

Tabla 7. Relación entre la capacidad coordinativa y la agilidad

\begin{tabular}{crcccc}
\hline Model & \multicolumn{2}{c}{$\begin{array}{l}\text { Unstandarized } \\
\text { Coefficients }\end{array}$} & $\begin{array}{c}\text { Standartized } \\
\text { Coefficients }\end{array}$ & & \\
& \multicolumn{1}{c}{$\mathrm{B}$} & Std. Error & Beta & T. & Sig. \\
\hline 1 (Constant) & 17.809 & 4.644 & & 3.835 & 0.001 \\
Agilidad & 0.059 & 0.252 & 0.45 & 0.236 & 0.815 \\
\hline
\end{tabular}

a. Dependent Variable. Cap_Coor_t

Fuente: elaboración propia.

Por otra parte, el tiempo promedio asociado con la agilidad (18.4223 $\pm 1.17790)$ es levemente inferior al de la capacidad coordinativa (18.9037 \pm 1.59467); sin embargo, al aplicar la prueba t-student (tabla 8), se determinó que la diferencia promedio entre los tiempos de ejecución del recorrido para la agilidad y la capacidad coordinativa, es no significativa (los tiempos promedio son similares), ya que su valor_p (Sig-tailed) $=0.189$ es superior al 0.05 .

Tabla 8. Prueba t-student para agilidad $v$ s. capacidad coordinativa

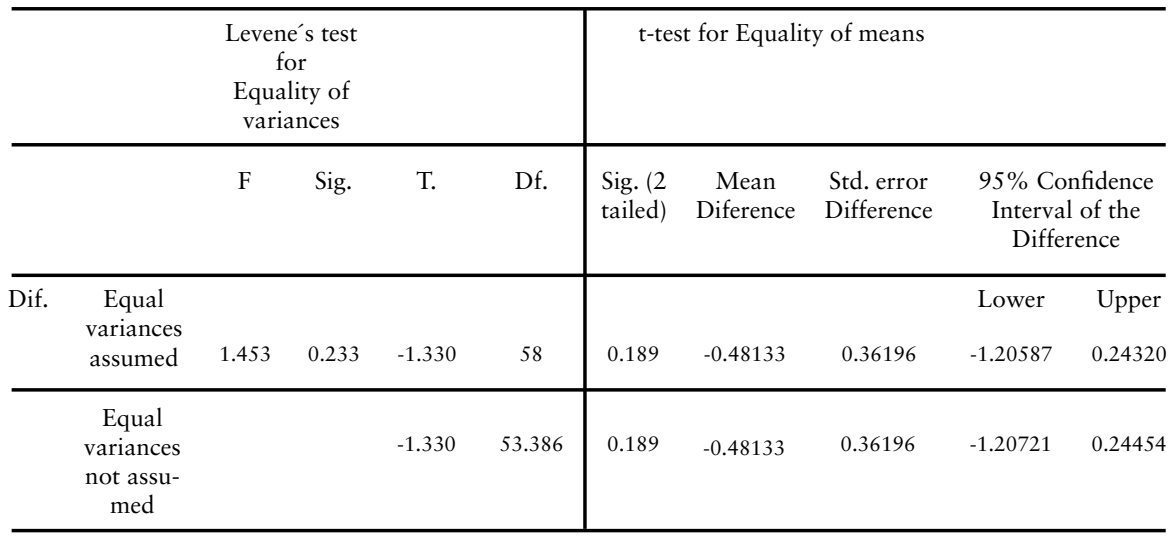

Fuente: elaboración propia. 
De los resultados obtenidos en el análisis correlacional respecto a la relación que existe entre la agilidad y las capacidades coordinativas en los niños de 12 a 14 años de edad que practican el fútbol en la escuela de formación deportiva del IRDET de Tunja, se comprobó que existe una relación no significativa, débil y directa entre la agilidad y la capacidad coordinativa, lo cual indica que un aumento en la agilidad implica un incremento débil en la capacidad coordinativa y viceversa, de los niños involucrados en esta investigación.

A pesar de que el tema objeto de aplicación carece de estudios similares o complementarios que analicen este tipo de población y el espacio donde ejecutan sus actividades, razón por la cual no se pueden comparar, confrontar o afirmar los resultados presentados por los autores relacionados en los antecedentes con los obtenidos en la presente investigación; se retoman algunas de sus conclusiones, donde Duncan (2014) reitera la importancia que tiene la agilidad en lo deportivo en el análisis teórico que hace; Moreno y Agudelo (2016), al estudiar la correlación entre fuerza explosiva y capacidades coordinativas, encuentran una relación entre baja y sin significación; Solana y Muñoz (2011) reafirman (sin comprobación estadística) la importancia del trabajo de las capacidades coordinativas en la formación de los jóvenes futbolistas y formulan una propuesta de entrenamiento; García, Pérez, Rodríguez y Moral (2013) retoman la relación de las capacidades coordinativas con la competencia motriz autopercibida en una muestra significativa de adolescentes, varones y mujeres, concluyendo que las capacidades coordinativas se relacionan directamente con la competencia motriz autopercibida; García, Ruiz y Latorre (2015) analizaron la influencia del puesto específico desarrollado en el terreno de juego por jóvenes futbolistas en la capacidad de salto, sprint, agilidad y velocidad de golpeo, concluyendo que durante la etapa de formación del futbolista, en este caso la adolescencia, el nivel de especialización de los mismos no es alto y, en consecuencia, su condición física no presenta unos rasgos marcados, específicos de una determinada posición en el campo, probablemente debido a que en estas edades y niveles, se persigue una formación integral del deportista y un desarrollo lo más amplio posible, tanto a nivel físico como técnico-táctico; Palma, Rosero y Dávila (2014) estudiaron el efecto de un programa de entrenamiento motriz sobre la agilidad y la coordinación de niños en edad escolar temprana, corroborando los fundamentos e investigaciones de los autores que 
tomaron ellos como referencia, comprobando que se mejora la motricidad de los estudiantes sometidos a la investigación, en especial, en lo referente a las capacidades relacionadas con el control y regulación del movimiento y Melo (2014) se propuso analizar los criterios en la evaluación de la agilidad para la selección de talentos en el fútbol base y, para ello, le da relevancia a la agilidad con criterios a tener en cuenta (evaluación cuantitativa y cualitativa), pero no muestra resultados concluyentes que se puedan confrontar con el presente estudio.

\section{Conclusiones}

Con estos resultados de la investigación, se da respuesta a los objetivos planteados en función de ver la relación existente entre la agilidad y la capacidad coordinativa, en niños de edades que fluctúan entre los 12 y los 14 años que practican el fútbol en la escuela de formación del IRDET.

Con fundamento en un análisis cuantitativo, se puede afirmar que el grupo de estudio resultó homogéneo en referencia a la agilidad con un tiempo promedio de recorrido de 18.422 segundos aproximadamente, que lo clasifica en el nivel promedio bajo, según la escala establecida en el test de Illinois; además, un alto porcentaje de los participantes logró clasificarse en el promedio o por debajo de este.

En lo referente a las capacidades coordinativas, se evidenció que el grupo de estudio fue homogéneo con un tiempo promedio de trayecto de 18.903 segundos aproximadamente, valor que lo clasifica en el nivel de bueno en concordancia con el test de Caminero (2009); adicionalmente, un alto porcentaje de los niños se clasificó en el nivel de bueno sobre la media, bueno, muy bueno o extremadamente bueno.

Con base en el análisis correlacional, se concluye que existe una relación no significativa, débil y directa entre la agilidad y la capacidad coordinativa, lo cual indica que un aumento en la agilidad implica un incremento débil en la capacidad coordinativa y viceversa.

Por otra parte, al comparar los tiempos promedio asociados tanto con la agilidad como con la capacidad coordinativa, el tiempo promedio para la agilidad es levemente inferior al de la capacidad coordinativa, 
aunque de forma no significativa, por lo que puede considerarse que tales tiempos son similares.

Con base en el análisis correlacional se concluye que existe una relación no significativa, débil y directa entre la agilidad y la capacidad coordinativa, lo cual indica que un aumento en la agilidad implica un incremento débil en la capacidad coordinativa y viceversa.

\section{Referencias}

Caminero, L. (2009). Diseño y estudio científico para la validación de un test motor original, que mida la coordinación motriz en alumnos/as de educación secundaria obligatoria. Granada, España: Universidad de Granada. DOI: https://doi. org/10.25145/c.educomp.2018.16.104

Duncan Mwirigi, M. (2014). La capacidad coordinativa agilidad: una atención temprana. Recuperado de: Revista digital de ciencias aplicadas al deporte, 6(14), 43-55.

García-Pinillos, F., Ruiz-Ariza, A. y Latorre-Román, P. Á. (2015). Influencia del puesto específico en la potencia y agilidad de jóvenes futbolistas. Retos, (27), 58-61. DOI: https://doi.org/10.1016/j.apunts.2014.05.002

García-Canto, E., Pérez, J. J., Rodríguez, P. L. y Moral, J. E. (2013). Relación de las capacidades coordinativas con la competencia motriz autopercibida en adolescentes. Trances, 5(3), 213-228. DOI: https://doi.org/10.1787/888933091790

Melo C., L. (2014). Análisis de la agilidad, mediante la aplicación de test físicos, en jugadores de futbol pre-juvenil, como criterio fundamental en la selección de talentos deportivos. Universidad Pedagógica de Colombia, Facultad de Educación Física. Programa de Especialización en Pedagogía del Entrenamiento Deportivo, Bogotá D. C., Colombia. DOI: https://doi.org/10.17533/udea. le.n $84 \mathrm{a} 05$

Moreno Rivera, I. y Agudelo Velásquez, C. (2016). Correlación entre fuerza y capacidades coordinativas en escolares del Liceo León de Greiff (Tunja, Colombia). VIREF Revista de Educación Física, 5(3), 18-26. Recuperado de: http://a prendeenlinea.udea.edu.co/revistas/index.php/viref/article/ viewFile/325965/20783262

Palma Pulido, L., Rosero Rosero, M. y Dávila Grisales, A. (2014). Efecto de un programa de entrenamiento motriz sobre la agilidad y las capacidades coordinativas 
en niño/as en edad escolar temprana. Revista de Educación Física, 30(2), 27-41. DOI: https://doi.org/10.15332/tg.mae.2017.00373

Pérez, P. (2016). Programa de preparación competitiva a entrenadores del departamento del Meta. Villavicencio, Colombia: Indeportes.

Solana, A. y Muñoz, A. (2011). Importancia del entrenamiento de las capacidades coordinativas en la formación de jóvenes futbolistas. Revista Internacional de Ciencias Sociales y Humanidades, SOCIOTAM, 21(2), 121-142. DOI: https:// doi.org/10.18848/2471-867x/cgp

Young, W. y Farrow, D. (2006). A review of agility: Practical applications for strength and conditioning. Strength and conditioning journal, 28(5), 24. DOI: https://doi.org/10.1519/00126548-200610000-00004 